\title{
A Pressure-Sensing Mouse Button for Multilevel Click and Drag
}

\author{
Masaki Omata ${ }^{1}$, Kenji Matsumura $^{2}$, and Atsumi Imamiya ${ }^{1}$ \\ ${ }^{1}$ University of Yamanashi, Interdisciplinary Graduate School of Medical and Engineering \\ ${ }^{2}$ University of Yamanashi, Department of Computer Science and Media Engineering, \\ Takeda 4-3-11, 400-8511 Kofu, Japan \\ \{omata, kng, imamiya\} @hci.media.yamanashi.ac.jp
}

\begin{abstract}
This paper proposes a pressure-sensing mouse button that can better express the user's intention. When users use it for click or drag, the users can specify continuous parameters of the mouse operation by adjusting intensity of the button press between from lightly to strongly. For evaluating it, we conducted experiments to validate optimal number of pressure levels and to compare the mouse with conventional input methods. Subjects required significantly more time with eight pressure levels than with fewer levels. We also found that the mouse was not faster than conventional mice in terms of task performance time because users were not adept at adjusting force of the mouse button. We, therefore, suggest that an effective operation for the pressuresensing mouse button is to change an insignificant effective parameter associated with fundamental determination of a mouse operation.
\end{abstract}

Keywords: Mouse button, pressure-sensing button, multilevel button press, pressure.

\section{Introduction}

The mouse, along with the keyboard, is the most popular computer input device. A mouse is typically used for pointing an object, selecting objects and deciding an operation in a GUI. When the user moves the mouse, a corresponding pointing cursor moves on the screen. When the user clicks a mouse button, the object on a screen being pointed to is selected.

With a conventional mouse, users can continuously adjust the position of the pointing cursor or of a scroll box by adjusting the distance the mouse is moved or by the rotation of a scroll wheel. However, with the conventional mouse button, the user can only choose the alternative of "selected" and "not selected." Whether the user clicks the mouse button lightly or heavily, there is no difference in the results.

With a mouse button that permits multilevel clicks through varying intensity of pressure, the user is able to specify a continuous parameter of an operation. Just as a pianist can express his or her intention through how hard he/she strikes the keys, we propose that multilevel clicks can better reflect the intentions of a computer user.

There have been previous studies examining multilevel mouse clicks and multilevel intensities of stylus pressure. Zeleznik et al. developed the "pop through" 
mouse button [1]. This button allows a user to click at two levels to control a property with a single button. Ramos et al. proposed GUIs that reflect pressure of a stylus to the state of windows, icons, menus and pointers (WIMP) [2]. Mizobuchi et al. suggested using stylus pressure in a handheld device to control a WIMP interface [3].

However, Zeleznik et al.'s pop-through button does not provide enough levels to reflect details in a user's intentions, while pressure-sensing styluses cannot be applied to most desktop computers. We, therefore, propose the "multilevel force-sensingbutton mouse" that distinguishes an intensity of pressure of a mouse button and reflects user's intention to results of an operation. This paper describes experiments on human characteristics with a pressure-sensing mouse button, compares its usability with other devices, and introduces the prototypes of applications.

\section{Related Work}

In this section, we introduce some related studies about multilevel input methods.

Ramos et al. suggested implications for the design of pressure-sensitive widgets [2]. They conducted an experiment investigating human characteristics in the use stylus pressure to perform discrete target acquisition tasks with different selection techniques. The results indicate that the quick release selection technique was preferable overall, and that dividing pressure space into six levels is optimal.

Ramos et al. also suggested the "Zliding" for fluid integrated manipulation of scale (zooming) via pressure input while parameter manipulation within that scale is achieved via $\mathrm{x}-\mathrm{y}$ cursor movement (sliding) [4]. By using this system, users can use the pressure modality to fluidly and explicitly zoom or adjust the granularity of the parameter space, while sliding or dragging the input device.

Mizobuchi et al. suggested a potential space saving technique that can be used for selecting menus, text/number input, changing windows, zooming/panning, etc. in penbased handheld devices [3]. They examined speed and accuracy of force input with a pen on a pressure-sensitive screen mounted on a handheld device. They reported that force for controlling a GUI are likely in the range 0 to $3 \mathrm{~N}$, and the number of levels within that range should be between five and seven.

Rekimoto et al. suggested the "PreSenseII" that recognizes position, touch and pressure of a user's finger [5]. This device acts as a normal touchpad, but also senses pressure for additional control. As the example applications of the device, they introduced map navigation that allows a user to scroll a map and to zoom in/out with sliding a finger and pressing the touchpad and a long item list that allows a user to control scrolling with pressing predefined area.

Blaskó et al. developed the "Pressure-Sensitive Strips" that allows one-handed direct control of a large number of parameters by detecting contact pressure differences of user's fingers [6].

While these studies used a stylus or a touchpad to input pressure, our study uses a mouse button, which is a more popular input device. We examine the human characteristics of a pressure-sensing button because we cannot use the previous results for a stylus. However, to design experiments for the pressure-sensing mouse button, we refer to the existing data on the human characteristics of controllable range of a stylus.

Zeleznik et al. developed a pop-through mouse buttons that achieve double-action effect by converting the standard mouse buttons to pop through tactile pushbuttons 
[1]. The system allows two levels of clicks, pressing lightly and pressing firmly. For example, pressing the button lightly could bring up a short menu, while pressing harder brings up a longer menu.

Forlines et al. presented a model for multilevel input devices based on a "glimpse state" [7]. By adding a glimpse state to a three-state pressure sensitive input device, user is able to preview the effects of their editing without committing to them. They suggested that the glimpse state has the added benefits that the negative effects of inconsistencies in the undo feature within an application are reduced.

Both of these studies proposed two levels of click operation with a mouse button. We propose that multilevel operations (over three states) can pick up more detail of a user's intentions. By pressing lightly or heavily, we suggest that the multilevel operations (click and drag) allow a user to input a value of properties, urgency, and his or her emotion. On the matter of emotion, Swindells et al. introduced that user interactions with tactile haptic feedback combine to strongly influence the user's emotional disposition and attitude [8]. On the matter of biometrics, Ikehara has developed a Pressure Mouse that can identify the user by the way he or she clicks it [9]. The mouse incorporates some electronic sensors to register hand pressure, speed and number of clicks exerted on the mouse's buttons.

Qi et al. mounted eight pressure sensors on a computer mouse to collect mouse pressure signals from users [10]. They suggested that the pressure mouse was used to classify two regions of each user's behavior: mouse pressure where the form-filling process is proceeding smoothly, and mouse pressure following a usability bug. This approach is based on the hypothesis that subjects apply more pressure to the mouse after encountering frustrating events. They suggested that user frustration could be measured by sensing the pressure applied to the mouse body. Our pressure-sensing mouse button, in contrast, reflects the user's intention to user's operation directly and concretely by sensing intensity of pressure while the user clicks the mouse button or drags the mouse to operate an application.

\section{Pressure-Sensing Mouse Button}

Fig. 1-(a) shows the prototype pressure-sensing mouse button that we developed. We mounted a commercially available pressure sensor (CUI IESP-12, Phidgets [11]; Fig. 1-(b)) on the left mouse button. It senses forces from 0 to $3 \mathrm{~kg}$ and generates a corresponding value from 0 to 1000 . Data from the pressure sensor are sent to the computer via USB. To make the sensor more comfortable, we covered it with a cap that we made out of clay to fit the index finger. For the human perception experiment, we used the sensor without the ergonomic cap because it was necessary to analyze human perception for the sensor itself. However, in the comparison with other devices we used the ergonomic cap to make the comparisons more fair.

Fig. 2 shows the use of the pressure-sensing mouse button. It can be used for pointing, clicking the right mouse button, and dragging it by pressing the right button, as with a conventional mouse. On the other hand, a user clicks the pressure-sensing button on the left for clicking it and dragging it. As the user presses the pressuresensing button, the underlying left button is pressed simultaneously, so the user can operate the left mouse button as easily as a normal mouse button. 


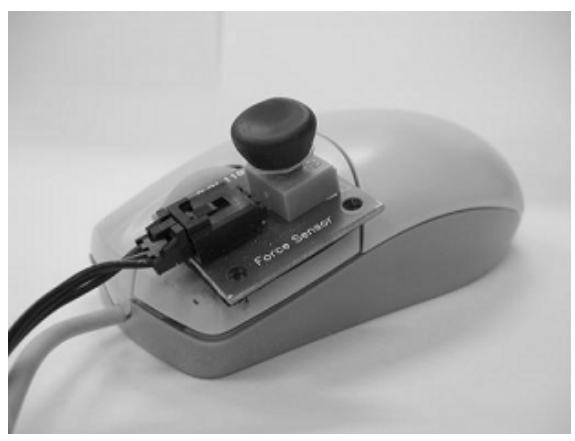

(a) The pressure-sensing mouse button

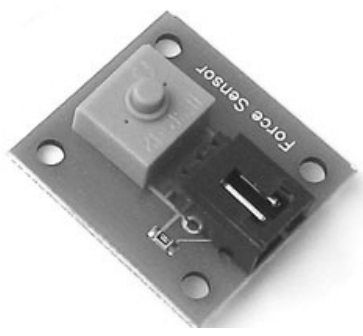

(b) pressure sensor [11]

Fig. 1. The pressure-sensing mouse button (a) and pressure sensor (b) [11]

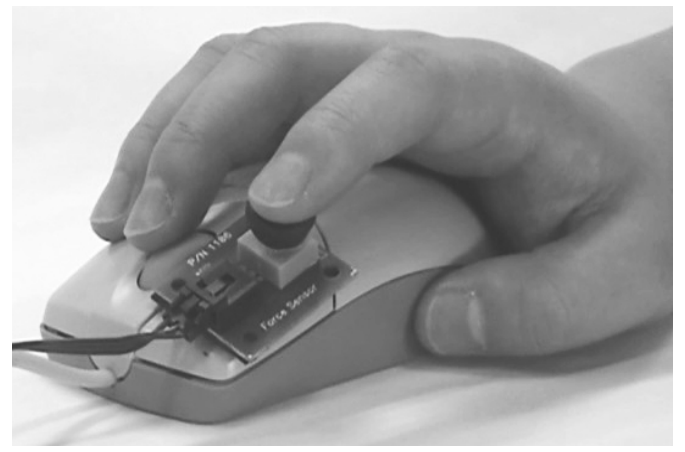

Fig. 2. Use of the pressure-sensing mouse button

\section{Prototypes of Applications: Pressure-Input Dynamic Map and Pressure-Jump Web Browser}

In this section, we suggest two applications using the pressure-sensing mouse button.

\subsection{Pressure-Input Dynamic Map}

The pressure-input dynamic map is a dynamic map controlled by the user's pressure input. The level of graphical and text detail increase with increasing pressure applied. For example, if a user wants to know information along the route to a station, the user follows the route with the pointing cursor by moving the mouse. To get greater detail about the area around the cursor, the user presses more heavily. Finally, the original map, which reflects the user's intentions, is created while the user follows the way on a map by dragging the pressure-sensing button.

Fig. 3 illustrates the system. The cells on the map are the minimum changeable areas. First, the system displays a default map, as in Fig. 3-(a). As the user drags the cursor up the street on the right while applying pressure, more detail about the route is revealed (Fig. 3-(a)). Finally, the user can get the original map to know the details of the main street. 


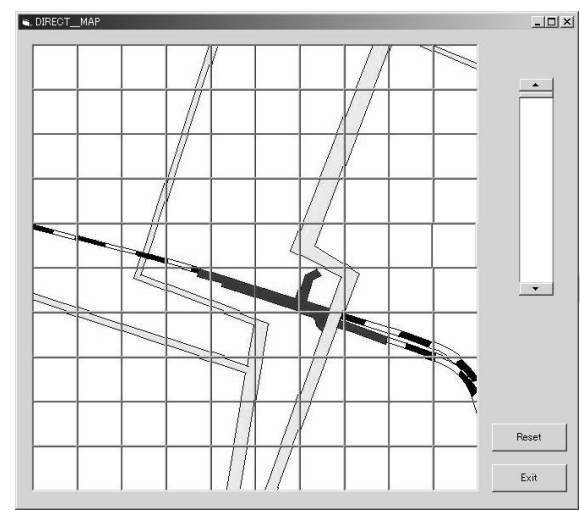

(a) Default map

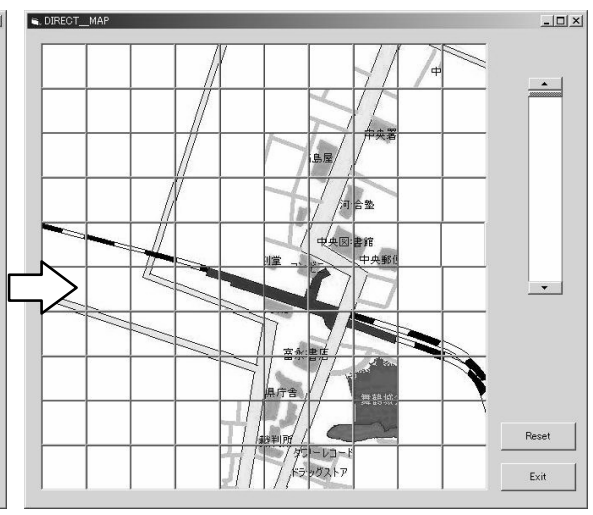

(b) User's map

Fig. 3. Pressure-input dynamic map. (a): Default map. (b): Map user creates by dragging along the route while applying pressure.

\subsection{Pressure-Jump Web Browser}

With a pressure-jump web browser, the pressure-sensing button can be used to control the amount of information displayed from a link (Fig. 4). Pressing the button heavily displays the full contents of the linked page (Fig. 4-(b)), while pressing lightly only displays the text of the linked page, eliminating pictures and animations (Fig. 4-(c)). Therefore, a user can adjust levels of the contents of a web page in his or her intentions.

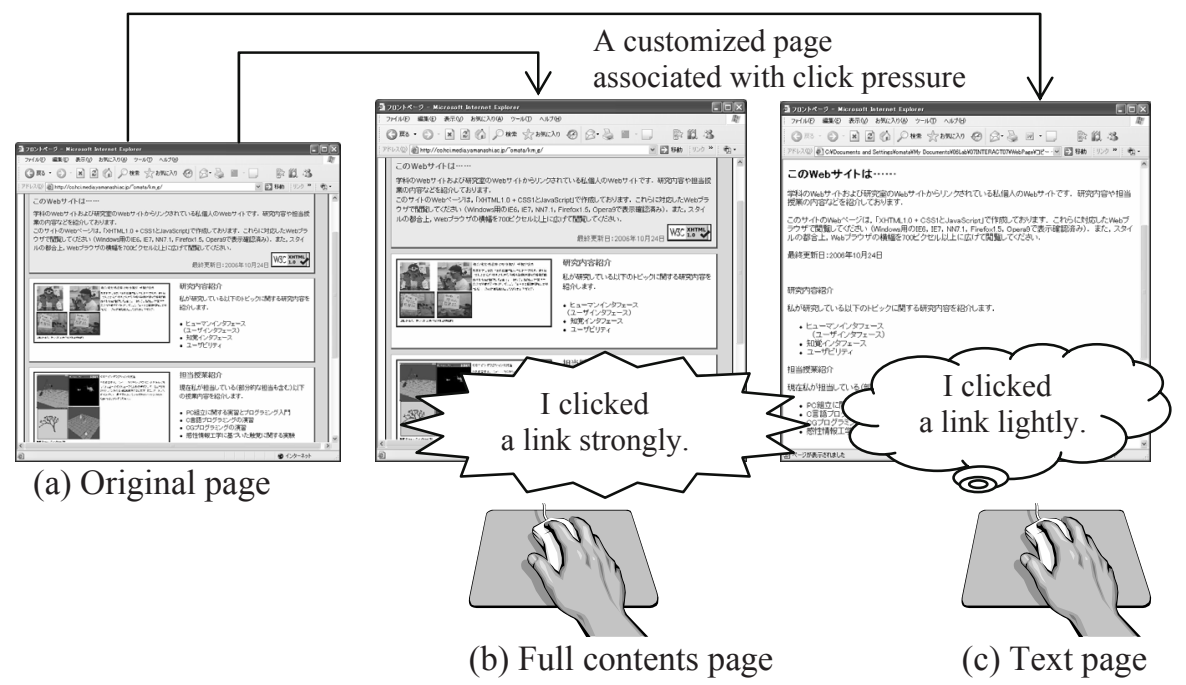

Fig. 4. A web browser using the pressure-sensing mouse button 
The prototype in Fig. 4 is a mock-up. The prototype displays only pre-created pages that we created for each level preliminarily. We plan to develop the application as a plug-in for a popular web browser.

\section{Human Characteristics of the Pressure-Sensing Mouse Button}

We conducted an experiment to validate the human operating characteristics of our pressure-sensing mouse button. We validated two characteristics:

- The optimal number of pressure levels

- The pressure determination method decision

\subsection{Optimal Number of Pressure Levels}

In this experiment, we validated the optimal number of pressure levels for inputting the user's intentions. The experimental factors were the number of partitions of pressure and preconditions. Based on Ramos et al.'s finding that the optimal number of pressure levels for stylus input is six, we compared four, six and eight pressure levels. We compared two conditions: "as fast as possible" and "as accurate as possible." The dependent variables were task performance time and number of errors.

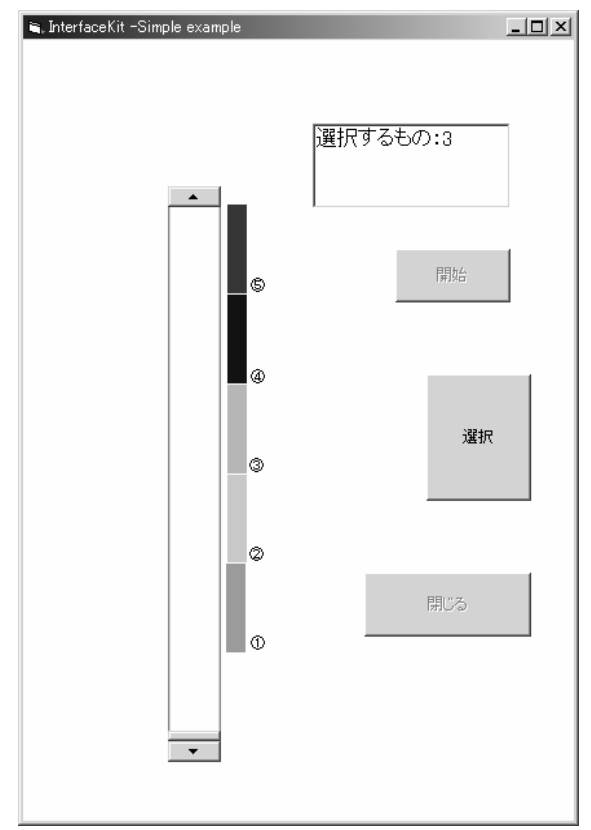

Fig. 5. Software to select a part of pressure partitions with the pressure-sensing mouse button

The experimental task is to select a part of pressure partitions and determine it by pressing the left button, adjusting the intensity of pressure of it, and clicking right mouse button. Subjects were presented with a target pressure level and asked to 
achieve it with the pressure-sensing button. Fig. 5 shows the software the subjects used to perform the task. A scroll bar labeled by both number and color is used to display the target levels, while the height of the scroll box reflects the pressure intensity applied to the button. Fig. 5 shows the case of six pressure levels; the lowest range is the play of pressure. On the right side of the screen, the upper and lower buttons start and finish, respectively, one block of trials, and the middle button is the home position for the pointing cursor.

The subject first depresses the pressure-sensing button and then clicks the middle button on the window. Next, a target level is presented in the text box and the subject adjusts the intensity of pressure to keep the scroll box in the target range. Finally, the user presses the right mouse button to confirm the intensity. For example, on Fig. 4, the target range number is " 3 " in the text box, so the subject should adjust pressure as the medium intensity.

The task performance time was measured as the time from clicking the home position button to clicking the right mouse button at the end of the task. An error is defined as when the range confirmed with the right mouse button is different from the target range.

Each subject performed all trials in all conditions: two preconditions, and three partition levels. There were 14 trials for each condition, so that each subject performed 84 trials $(2 \times 3 \times 14)$. All subjects were given the same set of target levels, but they were presented in random order.

After completing the trials, subjects answered a questionnaire about the pressuresensing mouse button (Table 1). The questionnaire consisted of eight questions about fatigue, difficulty of control and impressions of the mouse, each rated on a six-point Likert scale $(1=$ worst, 6 = best $)$.

Table 1. Questionnaire on pressure-sensing mouse button

\begin{tabular}{|c|c|}
\hline & $\begin{array}{l}\text { What do you think about feelings of fatigue in comparison with a usual mouse? } \\
\text { (very fatigued (1) - never fatigued (6)) }\end{array}$ \\
\hline & $\begin{array}{l}\text { How is it to associate an event with pressing the force button? } \\
\text { (very difficult (1) - very easy (6)) }\end{array}$ \\
\hline & $\begin{array}{l}\text { What do you think about the size of the scrollbar? } \\
\text { (very small (1) - very large (6)) }\end{array}$ \\
\hline & $\begin{array}{l}\text { How is it to learn how to confirm the intensity with right click? } \\
\text { (very difficult }(1) \text { - very easy (6)) }\end{array}$ \\
\hline & $\begin{array}{l}\text { What do you think about adjusting the intensity of pressure? } \\
\text { (very difficult (1) - very easy (6)) }\end{array}$ \\
\hline & $\begin{array}{l}\text { How is it to understand the system intuitively? } \\
\text { (very difficult (1) - very easy (6)) }\end{array}$ \\
\hline & $\begin{array}{l}\text { Do you prefer this system? } \\
\quad(\text { not at all }(1) \text { - very much }(6))\end{array}$ \\
\hline & $\begin{array}{l}\text { Are you interested in this system? } \\
\quad(\text { not at all (1) - very much (6)) }\end{array}$ \\
\hline
\end{tabular}


Twelve subjects (eight men and four women) in their twenties and thirties participated in this experiment. All were right handed and they typically used a computer between two to eight hours a day. The subjects practiced to use the pressure-sensing mouse button adequately before they performed an experimental task. Fig. 6 shows the average of task performance times of all subjects for each number of levels. Fig. 7 shows the average of error rate of all tasks for each number of levels. The solid line in the figures represents the results for the precondition "as fast as possible," and the dotted line represents the results for the precondition "as accurate as possible."

The results indicate that the subjects performed the task significantly faster in the precondition "as fast as possible" than in the precondition "as accurate as possible" (ANOVA: F $(1,11)=59.88, \mathrm{p}<.01)$. Moreover, the subjects required significantly more time with eight levels than with six or four levels (multiple comparisons among the partition levels: $\mathrm{p}<.01$ ).

The results indicate that the subjects performed the task significantly more accurately in the precondition "as accurate as possible" than in the precondition "as fast as possible" (ANOVA: $\mathrm{F}(1,11)=53.95, \mathrm{p}<.01$ ). However, there was no significant difference among the partition levels.

Fig. 8 shows a boxplot of the results of the questionnaire. In this figure, "+" corresponds to the median, the end-points of whiskers correspond to the maximum and minimum values, and the edges of the box correspond to the first and third quartiles. Most of subjects thought that the pressure-sensing mouse button was "easy to imagine the event," "easy to learn," "likable," and "interesting."

\subsection{Comparison of Pressure Determination Methods}

We redesigned and improved the determination method in the above experiment. In the new method, the subject maintains the intensity of pressure for $1 \mathrm{~s}$. The method allows users to adjust and confirm pressure using only the left mouse button. One

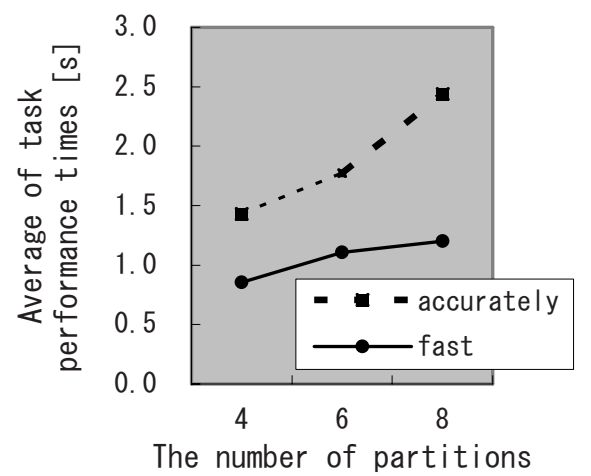

Fig. 6. Average performance times for pressure selection task

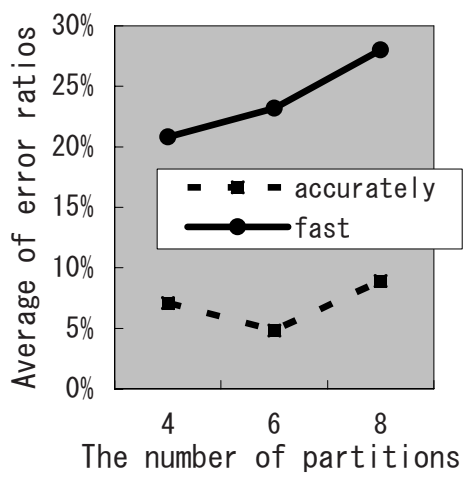

Fig. 7. Average error ratios for pressure selection task with the force-sensing mouse button 


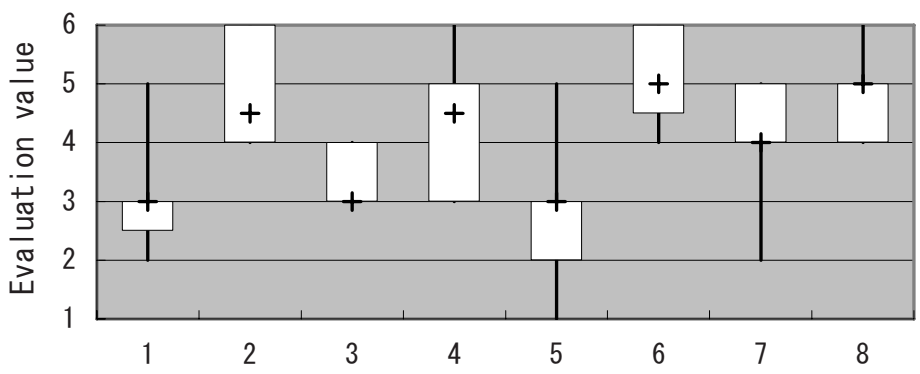

Fig. 8. Boxplot of questionnaire results

second was chosen after we contemplated it. In this experiment, we compared the determination method in the above experiment with the hold-for-1-s method by analyzing task performance time and error rate.

The task in this experiment was similar to the task in Section 5.1. The experimental factors were the precondition and the determination method, which was the main purpose. The precondition factors were similar to Section 5.1: "as fast as possible" and "as accurate as possible." There were two determination methods: maintaining the intensity of the pressure-sensing mouse button for $1 \mathrm{~s}$, and clicking the right mouse button while maintaining the intensity of the pressure-sensing button. Based on the results in Section 5.1, the number of pressure levels was fixed at seven. The seven levels included six selective ranges and a play range. Each subject performed 18 trials for each condition, so that the total number of trials for each subject was $72(2 \times 2 \times 18)$.

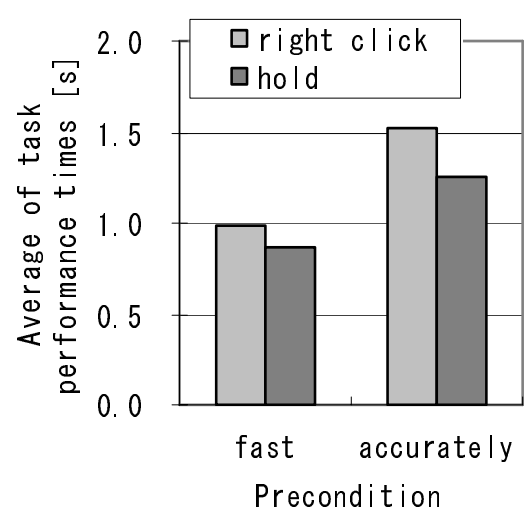

Fig. 9. Average of task performance times for comparison of determination methods

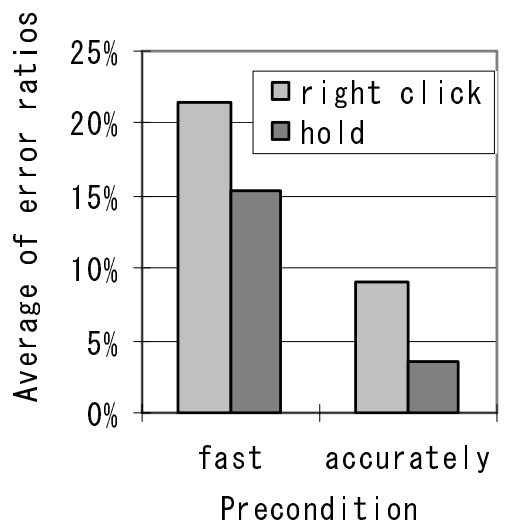

Fig. 10. Average of task error ratios for comparison of determination methods

Eight subjects, each of whom had participated in the previous experiment, participated in this experiment. Fig. 9 shows the average task performance time for each precondition, and Fig. 10 shows the average error rate for each determination 
method. According to ANOVA of the task performance times, there is a significant difference between preconditions $(\mathrm{F}(1,7)=95.37, \mathrm{p}<.01)$ and between intensity determination methods $(\mathrm{F}(1,7)=10.58, \mathrm{p}<.05)$. ANOVA of the error rate results showed that there is a significant difference between preconditions $(F(1,7)=15.34$, $\mathrm{p}<.01$ ), but not between intensity determination methods. The hold-for-1-s method resulted in a significantly shorter task performance time (ANOVA: $F(1,7)=10.58$, $\mathrm{p}<.05$ ), despite the requirement to maintain it for $1 \mathrm{~s}$. Moreover, using just the left mouse button better matches traditional click and drag operations, so the improved mouse adds the level of pressure input to current applications.

\section{Comparison with Conventional Input Methods}

Our next experiment compared the pressure-sensing mouse button with conventional input methods: moving the mouse and using a scroll wheel (Fig. 11).

The task of this experiment was to select a menu item using each of the input methods. Fig. 12 shows a menu bar from the experiment. The "START" part at the top of the menu is home position. The cross-hatched item is the current position of the subject's pointing cursor and the black item-whose position is random-is the target that the subject must place the cursor onto and keep it there for $0.5 \mathrm{~s}$.

For the mouse movement input, the subject clicks the right mouse button to bring up the menu and moves the pointing cursor by moving the mouse. For the scroll wheel input, the subject clicks the scroll wheel to bring up the menu and moves the pointing cursor by rolling the wheel. For the pressure-sensing button input, the subject presses the pressure-sensing button to bring up the menu and moves the pointing cursor by adjusting the intensity of the pressure; to select the target, the subject must maintain this intensity for $0.5 \mathrm{~s}$.

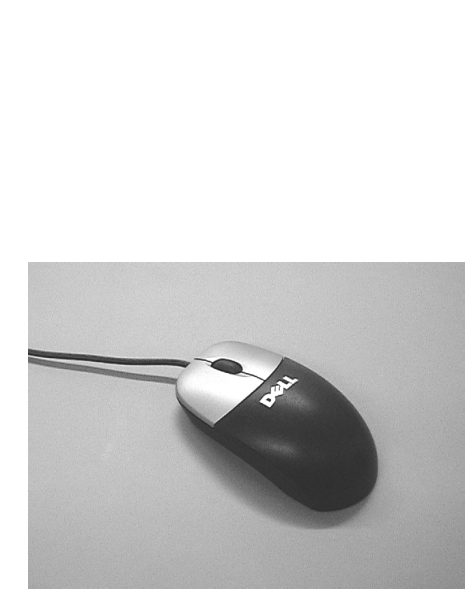

Fig. 11. A scroll wheel mouse (Dell Inc.) for the comparison experiment

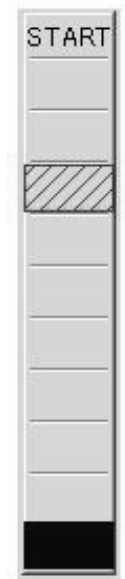

Fig. 12. Popup menu for the experimental task. The cross-hatched area is a cursor following the movement of the mouse. The black area is the target to which the curse is to be moved. 
The experimental factors are input methods and the number of partitions. For each input method, six different menu lengths were tested: five items, six items, seven items, eight items, nine items and 10 items. For each menu length, subjects performed 20 trials, so that each subject performed a total of 360 trials ( $3 \times 6 \times 20$ ). The dependent variable was task performance time.

Five subjects, all of whom participated in the human characteristics experiment in Section 5, participated in this experiment. Fig. 13 shows the average task performance time for each condition. ANOVA and multiple comparisons indicated that, for each menu length, the fastest input method was mouse movement and the slowest input method was the pressure-sensing button $(\mathrm{F}(2,8)=18.8, \mathrm{p}<.01)$. There was also a significant difference between five and nine menu items $(\mathrm{F}(5,20)=6.11, \mathrm{p}<.01)$.

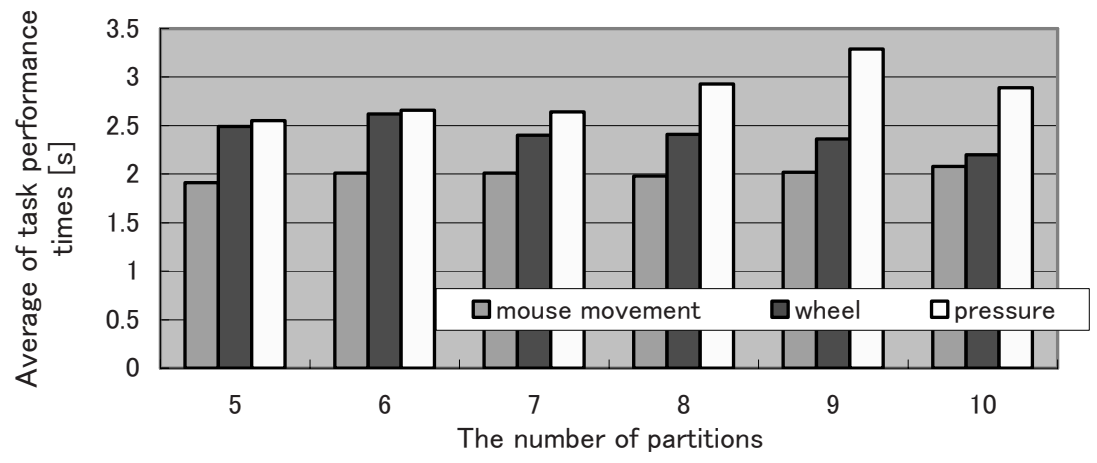

Fig. 13. Menu selection times for three input methods

One factor that made the pressure-sensing mouse slower than the other methods was the difficulty subjects had maintaining a constant pressure for the $0.5 \mathrm{~s}$ necessary to select a menu item. We observed that some subjects re-adjusted their intensity of pressure although they had already reached the correct intensity to hit the target.

\section{Conclusions}

The pressure-sensing mouse button expands the possibilities for a mouse button. The degree of freedom of a conventional button is one (pressed or not pressed), while in the pressure-sensing button there are multiple degrees of freedom, from pressing lightly to pressing heavily.

Our experiments showed that the pressure-sensing mouse button is inferior to conventional mice in task performance time. This is because subjects did not become as proficient in the pressure-sensing button as they were in the other devices. However, the scroll wheel was also once an unfamiliar device, but most users effectively use it today. We expect that the pressure-sensing mouse button will also be used efficiently with time, because subjects rated it as intuitive and easy to understand. 
There are several advantages of the pressure-sensing mouse button over the other input methods studied. It is easy for a user to control in several resolutions of intensity, such as five levels, six levels, and seven levels, because the maximum and minimum intensities are constant. It is easy for a user to return the input value to zero simply by removing the finger from the button. Moreover, the pressure-sensing button is suitable for inputting intentions of a user; pressing the button heavily corresponds to an intention to perform an operation quickly, in more detail or more emphatically.

However, there are disadvantages of the pressure-sensing mouse button over the other input methods studied. It is more difficult to select a level. It is particularly difficult for a user to maintain the intended pressure. Moreover, it is difficult to maintain pressure in the minimum range or in the maximum range.

We suggest that an effective application for the pressure-sensing mouse button is to change insignificant effective parameters associated with fundamental determination. For example, in clicking a "yes" or "no" button, the user can indicate the strength of his or her opinion with the force of the click. The user may even apply this intention pressure unconsciously. However, the user's choice of "yes" or "no" does not change.

We are planning to improve the hardware of the pressure-sensing mouse button by placing the pressure sensor under the mouse button. After that, we plan to explore useful applications for the mouse. An example of applications, pressing the pressuresensing button below the threshold for jumping to the linked page could present just a thumbnail of the page, with the size of the thumbnail corresponding to the level of pressure. As another example, the level of pressure could be used to set the priority for file downloads: pressing heavily would download the file as soon as possible, while clicking lightly would reduce the allocated bandwidth, preserving it for other uses.

Acknowledgments. We thank the members of the HCI group at the University of Yamanashi for their support of the research. This study was supported in part by the Grants-in-Aid for Scientific Research of the Japan Society for the Promotion of Science and by the RIEC of Tohoku University awarded to A. Imamiya.

\section{References}

1. Zeleznik, R., Miller, T., Forsberg, A.: Pop through mouse button interactions. In: Proceedings of the 14th annual ACM symposium on UIST 2001, Orlando, Florida, pp. 195-196. ACM Press, New York (2001)

2. Ramos, G., Boulos, M., Balakrishnan, R.: Pressure Widgets. In: Proceedings of the SIGCHI conference on Human factors in computing systems, Vienna, Austria, pp. 487494. ACM Press, New York, USA (2004)

3. Mizobuchi, S., Terasaki, S., Keski-Jaskari, T., Nousiinen, J., Ryynanen, M., Silfverberg, M.: Making an Impression: Force-Controlled Pen Input for Handheld Devices. In: CHI 2005 extended abstracts on Human factors in computing systems, Portland, OR, USA, pp. 1661-1664. ACM Press, New York, USA (2005)

4. Ramos, G., Balakrishnan, R.: Zliding: Fluid zooming and sliding for high precision parameter manipulation. In: Proceedings of the 18th annual ACM symposium on UIST 2005, Seattle, WA, USA, pp. 143-152. ACM Press, New York, USA (2005) 
5. Rekimoto, J., Schewsig, C.: PreSenseII: Bi-directional Touch and Pressure Sensing Interactions with Tactile Feedback. In: CHI 2006. extended abstracts on Human factors in computing systems, Montréal, Québec, Canada, pp. 1253-1258. ACM Press, New York, USA (2006)

6. Blaskó, G., Feiner, S.: Single-Handed Interaction Techniques for Multiple PressureSensitive Strips. In: CHI 2004 extended abstracts on Human factors in computing systems, Vienna, Austria, pp. 1461-1464. ACM Press, New York, USA (2004)

7. Forlines, C., Shen, C., Buxton, B.: Glimpse: a novel input model for multi-level devices. In: CHI 2005 extended abstracts on Human factors in computing systems, Portland, OR, USA, pp. 1375-1378. ACM Press, New York, USA (2005)

8. Swindells, C., MacLean, K.E., Booth, K.S., Meitner, M.: A Case-Study of Affect Measurement Tools for Physical User Interface Design. In: Proceedings of the 2006 conference on Graphics Interface, Quebec, Canada, pp. 243-250. Canadian Information Processing Society, Toronto, Canada (2006)

9. Malamalama Online Archive, Vol. 28, No. 1 (January 2003), http://www.hawaii.edu/ malamalama/archive/index.html, The magazine of the University of Hawai'i System, pp. 9-10, the last access date: (April 5, 2007) (2003)

10. Qi, Y., Reynolds, C., Picard, R.W.: The Bayes Point Machine for Computer-User Frustration Detection via PressureMouse. In: Proceedings of the 2001 workshop on Perceptive User Interfaces, Orlando, Florida, pp. 1-5. ACM Press, New York, USA (2001)

11. Phidgets, Canada (the last access date: April 5, 2007), http://www.phidgets.com/ 